Räumen zeigt: in jeder Alveole befindet sich gewöhnlich ein gut ausgebildetes Kriställchen in Form einer rhombischen Tafel, während rings um das Kristall, in einer breiten, leeren Zone, die Granula des Gels verschwinden, die eben zur Kristallbildung gedient haben ${ }^{3}$ ).

Schlieblich hat man aher eine wahre Lösung ohne Mizellen (Jodverbindung des Glvzerins), aber, bei geeigneter Konzentration, mit zahlreichen schwebenden und fixierten .Jodkriställchen.

Mit Terpentinöl bildet Jod ebenfalls eine gelbe Pseudolösung mit zahlreichen $\mathrm{Mj}$ zellén. Im gelben Lichte verbleicht diese Lösung nach und nach ohne Vermehrung der Mizellen ${ }^{4}$ ).

Die Lösung in Amylalkohol ist ebenfalls eine mizellare Pseudolösung, die aber sehr lichtempfindlich ist: durch die Belichtung erscheinen rasch Wolken von feinen Mizellen, welche sich nach und nach auf die Glasflächen feststellen. Bei Lichtabschlub findet eine teilweise Reversion dieser Mizellenbildung statt.

Die Lösung in Tereben bildet gewisse Eigentümlichkeiten. Das Jod scheint sich sehr

3) Dieselbe wabige Struktur des Gels sieht man bei Alcohol absolutus sehr deutlich nach Einwirkung des weißen Lichtes; doch kommt es hier nicht zur Kristallbildung, sondern nur zur Aggregation von Mizellenhaufen in der Mitte der Alveolen. haltig!

4) Das angewandte Terpentinol war stark ozon- rasch darin zu verflissigen, unter Bildung tiner eigentlichen Emulsion, deren Tröpfchen sich nach und nach auflösen Die dadurch gebildete l,osung ist ebenfalls sehr lichtempfindlich: es entstehen rasch Mizelletiwolken und die anfangs tiefbraune Lösung verbleicht nach und nach, um schließlich farblos zu werden.

Die Abhängigkeit der Intensitalt der Brownschen Bewrgung von der Zähigkeit der flüssigen Phase beobachtet man schr deutlich, einerseits bei der Pseudolösung in Essigäther, wo die Bewegung sehr lehhaft ist, andererscits bei Paraffinöl und Anvlalkohol, wo sie dagegen sehr träge, beinahe kaum sichtbar wird.

Es wären noch zahlreiche Eigentümlichkeiten zu beschreiben, weiche bei diesen Experimenten beobachtet wurden: so sind z. B. die Aetzungsfiguren der Jodkristallflächen, die man mi! den verschiedenen l.ösungsmitteln erhält, sehr merkwürdig and sehr verschieden, doch mub ich mich vorderband damit begnügen, zum Schlusse, das Berbatinnugsmaterial in der Form einer tabellarischen Jebersicht cier studierten Jodlösungen zusammanzustellen.

Fin Versuch, einige der beobachteten Erscheinungen in chemischer und physiko-chemischer Hinsicht zu deuten, soll einer späteren Arbeit vorbehalten werden.

Lausanne, im März 1910.

\title{
Bildung amikroskopischer Goldkeime durch Bestrahlung von Goldsalzlösungen mit ultraviolettem Licht.
}

Von The Svedberg.

(Ejngegaugen am 31. Mărz,
Durch einige im Zusammenhang mit meiner Untersuchung über Metallzerstäubung durch ultraviolettes Licht ${ }^{1}$ ) angestellten Versuche auf die große kondensierende Kraft der Lichtenergie aufmerksam gemacht, wurde ich ermutigt nachzusehen, in welchem Grade die Belichtung auf die Bildung einer kolloiden Lösung durch eine gewöhnliche chemische Kondensationsreaktion von EinfluB ist. DaB eine derartige Beeinflussung vorliegt, hat Mrne. Ful h a m e ) schon 1798 (Sonnenlicht), Küsperts 1902 und Vanin o 4) 1907 (Hg-Lampe) nachgewiesen

1) Diese Zeitschr. 6, 129-136 (1910).

7) Siehe in The Svedberg's "Herstellung kolloider Losungen* (Dresden 1909), S. 5 u. 14-15.

8) Bericht d. deutsch. chem. Ges. 35, 2815, 4066 , 4070 (1902)

4) Diese Zeitschr. 2, 51 (1907).
Wo. Ostwald gebührt der Verdienst auf die allgemeine Bedeutung des Lichtes als Kondensationsmittel zur Herstellung kolloider Lösungen aufmerksam gemacht zu haben.

Es wurde zunächst folgender Versuch angestellt. Eine mit Alkali versetzte sehr verdünnte Lösung von $\mathrm{HAuCl}_{4}$ wurde $30 \mathrm{~mm}$ unter die Heräus'sche $\mathrm{Hg}$-Lampe etwa $2 \mathrm{Min}$. bestrahlt und dann - noch helichtet .. mit Hydrazindichlorid reduziert. Die gebildete kolloide Lösung zeigte, im Uhtramikroskop betrachtet, einen amikroskopischen Lichtkegel.

Ein analoger Versuch ohne Belichtung gab eine Lossung mit gut sichtbaren, ziemlich großen Teilchen.

5) Grundris der Kolloidchemie (Dresden 1909), S. $303--304$. 
Die Versuche wurden mehrmals repetiert und immer mit gleichem Resultat: Diejenige loosung, welche bei Belichturg hergestellt war, enthielt immer viel kleinere Teilchen als die ohne Belichtang hergestellte.

Un diese Erscheinung näher kennen zu lernen, bestimmte ich unter verschiedenen Umständen mit und ohne Belichtung den Dispersitatsgrad der geblldeten kolloiden Lösungen. Die Anzahl Teilchen in einem bestimmten Volumen wurde mit Hilfe des SiedentopfZsigmondy'schen Ultramikroskops ermittelt.

A. Goldkonzentration konstant $=1 \cdot 10^{-4} \mathrm{Mol}$ $\mathrm{HAuCl}_{4}$ pro Liter. Reduziert wurde mit $\mathrm{N}_{2} \mathrm{H}_{4} .2 \mathrm{HCl}$, das bis zur Konzentration $1.10^{-3} \mathrm{Mol}$ pro Liter zugesetzt wurde.

Tabelle 1 .

\begin{tabular}{|c|c|c|c|}
\hline \multirow[b]{2}{*}{$\begin{array}{c}\mathrm{Mal} \\
\mathrm{NaOH} \\
\text { pro Liller }\end{array}$} & \multicolumn{3}{|c|}{ Teilchenrahl pro $384 \mu^{3}$} \\
\hline & $\begin{array}{l}\text { Bel Reduktion } \\
\text { oíne } \\
\text { Belirhtung }\end{array}$ & $\begin{array}{c}\text { Bei Rediation n } \\
\text { iang des } \\
\text { HAuCl + NaO } \\
100 \text { mm unter der } \\
\text { Hg - Lampe }\end{array}$ & $\begin{array}{l}\text { ach einer Belich- } \\
\text { Oenisches } \\
\text { von etwa } 2 \text { Min } \\
50 \text { mom inter der } \\
\text { Hg - Lampe } \\
\end{array}$ \\
\hline 0 & 1,0 & 6,8 & 10,0 \\
\hline $5.10^{-3}$ & 1,1 & $\cdots$ & \\
\hline $1.10^{-4}$ & 1,2 & 6,3 & 12,8 \\
\hline $1,2.10^{-4}$ & 1,4 & & $\cdots$ \\
\hline $1,5 \cdot 10^{-4}$ & 4,9 & 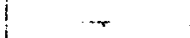 & $\ldots . .$. \\
\hline $2.10^{-4}$ & 6,8 & 5,7 & 12,10 \\
\hline $3,3.10^{-4}$ & 1,2 & $\ldots$ & --- \\
\hline $5.10^{-4}$ & Eine Mischung & - & $\cdots$ \\
\hline $8.10^{-4}$ & $\begin{array}{l}\text { ron weir } \\
\text { glefichero }\end{array}$ & 12,0 & - \\
\hline $1.10^{-3}$ & Teilch & -. & $-\cdots$ \\
\hline
\end{tabular}

Tabelle 2

\begin{tabular}{|c|c|c|}
\hline \multirow[b]{2}{*}{$\begin{array}{c}\text { Mol } \\
\mathrm{K}_{2} \mathrm{CO}_{2} \\
\text { pro Liter }\end{array}$} & \multicolumn{2}{|c|}{ Tellchenzahl pro $384 \mu^{3}$} \\
\hline & $\begin{array}{c}\text { Bei Reduktion } \\
\text { ohne Butichtung }\end{array}$ & 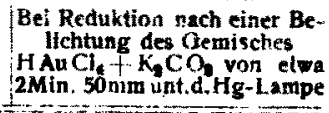 \\
\hline 0 & $1 ; 0$ & 10,0 \\
\hline $1.10^{-4}$ & 1,3 & 8,4 \\
\hline $2,5 \cdot 10^{-4}$ & 1,6 & 10,8 \\
\hline $3.10^{-4}$ & $\llbracket .2$ & 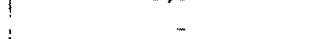 \\
\hline $4.10^{-t}$ & 5,6 & 10,8 \\
\hline $5.10^{-4}$ & $10 ; 2$ & $\ldots$ \\
\hline $1.10^{-3}$ & $11,5 ? \begin{array}{l}\text { Teilchen nicht } \\
\text { gleichgrof }\end{array}$ & 14,1 \\
\hline $\begin{array}{l}5.10^{-3} \\
1.10^{-2}\end{array}$ & $\begin{array}{l}\text { Yeil hen nichit } \\
\text { giefrhgrob } \\
5.6\end{array}$ & $\begin{array}{l}\text { Teilchen etwa } 20 u \mu \\
\text { sehr gleichgro }\end{array}$ \\
\hline $2.10^{-2}$ & 3,2 & $\int^{\text {Teilchen efras gröher als }}$ \\
\hline $3.10^{-2}$ & $-\cdots$ & 5,6 \\
\hline $4.10^{-2}$ & - & 1,2 \\
\hline $5.10^{-2}$ & 3,0 & 0,1 \\
\hline
\end{tabular}
B.

$$
\begin{aligned}
& \mathrm{HAuCl}_{4}=1 \\
& \mathrm{~K}_{2} \mathrm{CO}_{3}=5
\end{aligned}
$$

Variiert wurde die $\mathrm{Konz}$ des $\mathrm{HAuCl}_{4}$ bei Ausführung der Reduktion.

\begin{tabular}{|c|c|c|c|}
\hline \multirow{2}{*}{$\begin{array}{c}\text { Mol } \\
\text { HAuCle } \\
\text { pro liter }\end{array}$} & \multicolumn{3}{|c|}{$\begin{array}{l}\text { Teilchenzahl pro } 384 \mu^{3} \text { (auf die Konz. } \\
1.10^{-4} \text { normal } \mathrm{HAuCl}_{4} \text { zurtickberechnet) }\end{array}$} \\
\hline & $\begin{array}{c}\mathrm{Mol} \\
\mathrm{N}_{2} \mathrm{H}_{4} \cdot 2 \mathrm{HCl}\end{array}$ & $\begin{array}{c}\text { Bei } \\
\text { Reduktion } \\
\text { phne } \\
\text { Belichtung }\end{array}$ & 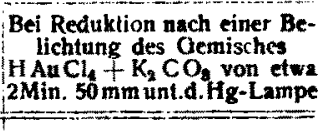 \\
\hline $\begin{array}{l}1.10^{-3} \\
2.10^{-4} \\
1.10^{-4} \\
\text { 1. } 10^{-5}\end{array}$ & $\begin{array}{l}1.10^{-2} \\
1.10^{-3} \\
1 \cdot 10^{-3} \\
1.10^{-3}\end{array}$ & $\begin{array}{l}0,34 \\
4,1 \\
10,2 \\
18,0\end{array}$ & $\begin{array}{c}0,24 \\
5,3 \\
12,0 \\
\text { Teilchen } 15-20 \mu \mu\end{array}$ \\
\hline
\end{tabular}

Bei sämtlichen dieser Versuche wurde das Reduktionsmittel noch während der Belichtung zugefügt. Es zeigte sich jedoch, daf dies nicht notwendig ist. Der gleiche Effekt stellt sich ebensogut ein, wenn die Goldsalzlösung zuerst belichtet und dann ohne Belichtung mit dem Reduktionsmittel versetyt wird.

Der Dispersitätsgrad der gebildeten kolloiden Lösung ist aber von der Dauer der Belichtung abhängig. Folgende Messungsreihe zeigt diese Abhängigkeit.

C. Goldkonzentration konstant $=1 \cdot 10^{-4} \mathrm{Mol}$ $\mathrm{HAuCl}_{4}$ pro Liter. Alkaligehalt $=0$. Reduziert wurde unmittelbar nach der Belichtung (jedoch ohne Belichtung bei Zusatz des Reduktionsmittels) mit $\mathrm{N}_{2} \mathrm{H}_{4} .2 \mathrm{HCl}$ bis zu $1.10^{-3}$ Mol pro Liter.

Abstand von der $\mathrm{Hg}$-Lampe $50 \mathrm{~mm}$.

\begin{tabular}{c|c}
\hline $\begin{array}{c}\text { Belichtungszeit in } \\
\text { Sekunden }\end{array}$ & Telichenzahl pro $384 \mu^{3}$ \\
\hline 5 & 1,1 \\
10 & 2,3 \\
60 & 5,3 \\
120 & 9,4
\end{tabular}

Bei noch längeren Belichtungszeiten wurde das Gold sichtbar ausreduziert. Beistehendes Diagramm gibt die Beziehung zwischen Teilchenzahl und Belichtungszeit graphisch wieder. Es ergibt sich eine fast gerade Linie.

Die durch eine gewisse Belichtung hervorgerufene Veränderung in der $\mathrm{HAuCl}_{4}-$ lösung verschwindet von selbst nur sebr langsam, denn eine $1.10^{-4}$ normal $\mathrm{HAu} \mathrm{Cl}_{4}$ - Lösung, die $120 \mathrm{Se}$ kunden mit der $\mathrm{Hg}$-Lampe belichtet wurde und dann 40 Min. im Dunkeln aufbewahrt wurde, gab bei der Reduktion 7,0 Teilchen pro $384 \mu^{3}$ statt der oben gefundenen Zahl 9,4. 


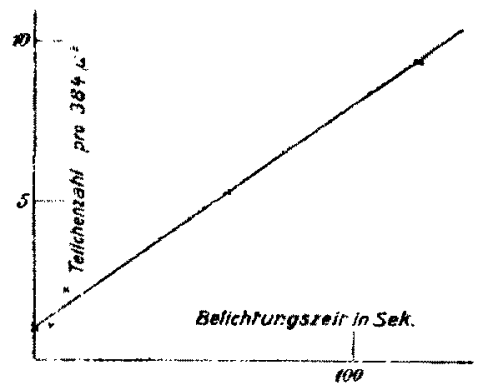

Die obigen Resultate können meines Erachtens nur folgenderweise erklärt werden.

Durcle das ultraviolette Licht wird eine Anzahi Goldmoleküle ausreduziert. Dieselben verejuigen sich zu Goldteilchen, welche zunächst amikroskopisch bleiben. Bei Zusatz des Reduktionsmittels wird eine stark übersättigte Goldmoleküllösung gebildet, auf der die Goldteilchen als Keime wirken, so daß das neureduzierte Gold sich hauptsächlich auf dieselben ablagert. Für diese Erklärung spricht der Umstand, daB die Teilchenzahl der Belichtungszeit proportional gefunden wurde, und ferner die Tatsache, dabs die durch die Belichtung hervorgerufene Veränderung in der Goldsalzlösung nur langsam verschwindet. AuBerdem konnte ich, wenn ich nach dem von Zsigmondy ${ }^{6}$ ) angegebenen Prinzip fertige amikroskopische Goldlösung zur $\mathrm{HAuCl}_{4}$-Lösung vor dem $\mathrm{Zu}$. satz des Reduktionsmittels zufügte, auch ohne Belichtung ganz dieselbe Erscheinung hervorrufen.

Die oben mitgeteilten Versuche sind für den Photochemiker von Interesse, weil sie die Existenz und Bedeutung von Amikronen in belichteten Metallsalzen beweist und eine sehr einfache Beziehung - nämlich direkte Proportionalität zwischen Belichtungszeit und Anzahl der gebildeten Keime -... anzeigt. Es kommt denselben auch eine gewisse methodische Bedeutung $z u$, denn es gelingt, nach diesem Prinzip wie die Messungsreihe $C$ es zeigt - eine gewisse Goldmenge innerhalb desselben Volumens mit verschiedenem Dispersitätsgrad zu zerteilen, ohne dab dazu verschiedene Zusälze eines Fremdstoffes nötig sind, wie z. B. bei Variierung des Dispersitätsgrades durch verschiedene Alkalizusätze. Dazu kommt noch der Umstand, daß die nach dieser Methode gewonnenen Goldsole aus sehr gleichgroßen Teilchen bestehen, was bei genaueren Messungen sehr wertvoll ist.

März 1910.

Chemisches Universitätslaboratorium Upsala.

6) Zeitschr. f. phys. Chem. 66, 65-76 (1906).

\title{
Die Wirkung des destillierten Wassers auf Metalle.
}

\author{
Von Margherita Traube-Mengarini and Alberto Scala.
}

(Eingegangen am 19. Mărz 1910)

Nachdem wir in früheren Abhandlungen 1) die Wirkung des kochenden destillierten Wassers auf Silber und Platin nachgewiesen haben, welche diese in kolloide Lösung überführt, behandeln diese Versuche die Löslichkeit des Bleis in destilliertem Wasser bei Stubentemperatur.

Dies Thema ist bereits in einer vielbändigen Spezialliteratur behandelt worden, aber so viel wir wissen, bis jetzt nur vom Standpunkte des Hygienikers aus. Der Hygieniker und durch ihn vor allem die englische Regierung, wollte untersuchen, unter welchen Umständen das Trinkwasser in Bleiröhren giftig würde, das heiBt also Blei löste. Merkwürdigerweise waren die Resultate dieser einfachen Versuche nicht übereinstimmend. Die Forscher blieben uneins, ob destilliertes Wasser Blei löse oder nicht. Diese

s) Rendiconti d. R. Acc. d. Lincei, 16 maggio e 15 agosto 1909. Atti d. Societa Italiana p. il progresso d. scienze 1910. Koll.-Zeitschr. 6, 65 (1910).
Versuche wurden unter vielfach abgeänderten Umständen gemacht. Die von Antony und Benelli $i^{2}$ ), welche fanden, daB sich Blei in destilliertem Wasser in Anwesenheit von Luft und ohne Luft löst, kamen dem Tatbestande am nächsten. Das heiBt, sie hatten insofern recht, als das Biei in Anwesenheit sowohl als in Abwesenheit von Luft vom destillierten Wasser angegriffen wird. In das, was man bis jetzt Lösung nannte, wird es aber uberhaupt nicht übergeführt. Das geht aus folgenden eintachen Versuchen zur Genüge hervor: Wenn man ein Stück Blei in destilliertes Wasser legt, es nach einiger Zeit herausnimmt und abwartet, bis das getrübte Wasser wieder klar geworden ist, und dann vorsichtig das Wasser von dem weiBen Bodensatz abgiebt, so findet sich auch nicht eine Spur von Blei in dem Wasser. Dies und ein anderer Umstand, den wir sofort erläutern

2) Gazzetta chimica ital. 26, Parte IIa, 353. 